\title{
Optimal Contracts under Moral Hazard and Costly Lying*
}

\author{
Keeyoung Rhee**
}

\begin{abstract}
We present a model in which the agent reports a privately observed signal about the stochastic outcome of her action, while bearing a cost of misreporting her private information. If the agent receives a low payment contingent on her performance, it is very costly for the agent to misreport her information to the principal so that the principal makes a decision favorable to the agent. However, if the contingent compensation is too high, the principal will terminate the project unless the agent truthfully reports that the project is likely to give a high return. The optimal outcome is achieved by a contract with the fee structure loosely tied with the outcome, but the cost of lying is necessarily high.
\end{abstract}

JEL Classification: D83, D86

Keywords: Contract theory, moral hazard, strategic communication, lying costs

\section{Introduction}

In a standard moral hazard problem, a principal is assumed to evaluate performance of an agent based on publicly observed outcome, which is an informative signal about the agent's action. However, many real-world observations suggest that such a signal is not always publicly observable to the principal. Instead, as Roger (2013) points out, agents report privately observed performance-related information to principals, which gives rise to a strategic communication problem $\grave{a}$ la Crawford and Sobel (1982) in addition to moral hazard. For instance, fund managers such as private equity firms (PEFs) review valuation of invested financial

Received: Feb. 13, 2020. Revised: July 3, 2020. Accepted: Nov. 30, 2020.

* This paper is a revised and translated version of Rhee (2019). The views expressed in this paper are those of the author and do not necessarily represent that of Korea Development Institute (KDI). The author is grateful to Sookyoung Yang for her able research assistance. All errors belong to the author. For expositional convenience, we throughout refer an agent to the female pronoun ("she") and a principal to the male pronoun ("he"), respectively.

* Associate Research Fellow, Department of Markets and Institutions, Korea Development Institute (KDI), Namsejong-ro 263, Sejong City, 30149, Korea, E-mail: ky.rhee829@gmail.com 
assets held by funds under their management prior to disclosing to their clients. This process, however, creates a possibility that fund managers, to their own favor, artificially inflate the value of the invested assets to make the investors stay confident and thus not request liquidation of the funds. Due to the shortage of information and expertise, investors can hardly detect the fund managers' misconduct in valuation until the funds fail to give returns at maturity dates.

There are legal (and social) systems to address such an incentive misalignment problem in information transmission by making any attempt of sending false information ("lying") costly. For instance, supervisory authorities impose penalties on the fund managers responsible for their wronging in valuation for invested assets. Furthermore, such a misconduct, once it becomes publicly known, will severely damage the manager' reputation in the financial markets, lowering their future income.

Nevertheless, recent cases of valuation frauds by PEF managers suggest that these costs of "lying" alone cannot fully resolve the incentive misalignment problem in information transmission. In April 11 2019, the Abraaj Group, a Dubai-based private equity firm, was charged by US Securities and Exchange Commission (SEC) with its misappropriation of money from the Abraaj Growth Markets Health Fund. According to the SEC's complaint, Abraaj misused the investors' money to cover its operating expenses, while inflating the value of the assets in its holding by more than hundreds of millions of dollars to deceive its clients. ${ }^{1}$ More recently, a Koreabased firm Lime Asset Management is facing multiple charges with mismanagement of its private equity funds, including the allegation of concealing poor performance of one its funds named Pluto TF-1.,3

These observations give rise to the following research questions: (i) how does a cost of "lying" influence the agent's incentive to (mis-)report her private information about the outcome? (ii) how should an optimal contract be designed to address both ex-ante moral hazard and ex-post strategic information transmission? Answering these questions will help us address real-world moral hazard problems more relevantly.

To answer the questions, we present a simple model which applies a costly lying framework à la Kartik (2009) to a moral hazard problem. In our three-date model, a risk-neutral principal delegates a task of managing a project to a risk-neutral agent at date 0 . The principal offers a contract consisting of a fixed-term upfront payment and a bonus payment contingent on the return of the project. The project gives a

\footnotetext{
${ }^{1}$ Go to https://www.sec.gov/litigation/litreleases/2019/lr24449.htm for more details.

${ }^{2}$ Go to https://www.koreatimes.co.kr/www/nation/2020/04/251_288438.html for more details.

3 Perhaps one of the most famous cases of financial statement manipulation is the Enron scandal (Healy and Palepu, 2003): the former CEO Jeff Skilling was sentenced to more than 24 years and fined $\$ 45$ million in 2006 for his commitment of corporate crimes, including making false statement to auditors.
} 
binary stochastic return: the project always gives high return if the return is realized at date 1 ; if not, the project gives zero or high return at date 2 .

The agent has an expertise in the project management on two aspects. First, by putting a costly effort at date 0 , the agent can increase the probability that the project gives high return at date 1 . Second, given that the project does not give return at date 1 , the agent observes an informative interim signal $s \in\{0, R\}$ about the project's future return. The agent reports her observed signal to the principal by sending a message $m \in\{0, R\}$. After observing the agent's report, the principal decides whether to continue the contract until date 2 or terminate the contract with the agent and select an outside option.

A key assumption is that there are two elements of information asymmetries between the principal and the agent in our model. First, the principal cannot observe the action's effort decision made at date 0 , which gives rise to an ex-ante moral hazard problem. Second, we assume that the agent privately observes additional information $s$, which gives rise to an ex-post strategic information transmission problem as Crawford and Sobel (1982). In addition, we further assume that the agent suffers from the cost of lying $L$ if she reports a message different from her observed signal (i.e., $m \neq s$ ).

We study how a compensation structure affects strategic interaction between the principal and the agent. To this end, we first characterize a date-1 partial equilibrium in which the agent, after observing $s=0$, pretends to observe the other signal by randomizing in sending the message $m=R$.

We find that the agent's date-1 equilibrium strategy — the probability of reporting $m=R$ after observing $s=0 \quad$ is non-monotonic to the bonus payment. To see why, suppose first the bonus payment is very low. If the agent misreports her information, she may successfully convince the principal to continue the project until $t=2$. However, since the expected payoff from managing the project until $t=2$ is lower than the cost of lying, the agent would rather report her private information truthfully.

Suppose next the bonus payment sufficiently high so that the expected payoff from misreporting exceeds the cost of lying. Then the principal expects a low expected net revenue because he has to yield a large share of the return to the agent. Hence, the agent will not always misreport her private information because such a strategy will convince the principal that she is lying, thereby leading to termination of the contract. By contrast, truthful reporting cannot be an equilibrium strategy, either. This is because the agent with $s=0$ will surely deviate and misreport her observed signal if the principal always continue the contract. Therefore, there exists a unique mixed-strategy equilibrium, where the agent with $s=0$ randomizes in sending the message $m=R$ and the principal randomizes in continuing the contract after receiving $m=R$. Furthermore, there is a threshold such that the probability that the agent sends $m=R$ after observing $s=0$ jumps from zero if 
the bonus payment exceeds the threshold. However, the probability that the agent misreports her signal is decreasing in the bonus payment as it exceeds the threshold: the agent's message must be more convincing as the principal's payoff from continuing the contract is decreasing.

This analytical result reveals how the bonus payment (adversely) influences the communicational inefficiency at date 1 . If the bonus payments increases, the principal will not continue the contract unless the agent is likely to send the message truthfully. This leads the agent with $s=0$ to send $m=R$ less likely in equilibrium. However, the principal will get a lower net expected payoff from misreporting as he has to yield a higher bonus payment. Thus, the principal will become less likely to continue the contract after receiving $m=R$ in order to incentivize the agent not to misreport her private information with a high likelihood. That is, when the compensation scheme is overly contingent on the project return, the principal uses his continuation decision as an incentive scheme of truthful reporting.

Lastly, we find an optimal contract to address moral hazard when lying is costly. Specifically, we find that a contract with a small bonus payment can implement the efficiency when the cost of lying is sufficiently high. As in a standard moral hazard problem, any optimal contract necessarily contains a bonus payment high enough to provide the agent with an incentive to make her effort on project management. In our setup, however, the principal must also take into account the ex-post consequence on the information transmission. Our paper proposes that the principal can resolve the ex-post inefficiency by offering a contract consisting of a bonus payment low enough to reduce the agent's incentive to misreport her information. Thus, the principal can resolve both incentive problems when the cost of lying is high: if not, any bonus payment addressing the ex-ante moral hazard problem inevitably admits ex-post misreporting, and vice versa. Given a low bonus payment, the principal must compensate the agent for forgoing her outside option when she accepts the principal's offer. As a consequence, the optimal contract offer becomes insensitive to the project return.

\section{Related Literature}

Economists have recently investigated how strategic information disclosure influences incentive structures of principals and agents in various frameworks. For example, in the auction literature, several papers such as Bergemann and Pesendorfer (2007), Eső and Szentes (2007), and Ganuza and Penalva (2010) analyze how sellers can increase their revenue by releasing additional information to bidders in various environments. They also study what the optimal information 
disclosure rules are. In the literature of organizational economics, papers such as Faure-Grimaud et al. (2003) study whether communication channels created by organizational structures can affect an incentive of agents to collude with each other.

Our paper analyzes how the moral hazard problem will be reshaped if an agent can strategically send her private information on her task to a principal. There are a number of papers studying the similar topics, such as Corgnet and Rodriguez-Lara (2013) and Roger (2013). The most related and frontier research is Roger (2013). In his paper, Roger (2013) presents a model in which the agent costlessly reports soft information on a delegated task. A key assumption in his model is that the principal can terminate the delegation contract after imperfectly verifying the agent's information at a cost. The main research question of Roger (2013) is that a remuneration scheme designed to resolve the ex-ante moral hazard problem adversely gives rise to a bias against the principal's termination decision, leading to a cheap talk problem. His main finding is that any contract cannot yield full information revelation by the agent.

Our paper departs from the same motivation as Roger (2013), but provides additional contributions to the existing literature with different scopes. First, and most importantly, we assume that the agent will suffer from a cost if she "lies" i.e., misreports her private information - to the principal. Such a lying cost is introduced to analyze how the development of auditing system influences the agent's incentive to truthfully report her information. Second, we assume that the principal lacks skills to verify the agent's message. Using this approach, we highlight an important role of the base payment in compensation structures which are not contingent on stochastic return of the project managed by the agent. Specifically, we find that an optimal contract is the one insensitive to the outcomes. Furthermore, we argue that the incomplete information problem with moral hazard and strategic communication can be resolved only if the auditing system is well-established.

This paper proceeds as follows. Section 3 introduces the model and discusses its relevance to the real world. Section 4 establishes equilibria and characterizes an optimal contract to resolve incentive misalignments. Section 5 concludes the paper. All proofs are relegated to Appendix A and B.

\section{Model}

\subsection{Setup}

Consider a three-date economy $(t=0,1,2)$ of a principal and an agent. Neither the principal or the agent discounts future payoffs realized in $t=1,2$. The principal owns a project $\tilde{R}$, which will generate a binary stochastic return in either 
$t=1$ or $t=2$. Specifically, the project gives a positive return $(\tilde{R}=R)$ with probability $p \in(0,1)$ in $t=1$, or the return of $\tilde{R}$ is delayed until $t=2$ with probability $1-p$. If the project returns in $t=2$, it is equally likely that the project yields either $\tilde{R}=R$ or $\tilde{R}=0$. Figure 1 depicts the stochastic structure of the project return. ${ }^{4}$

At date 0 , the principal delegates the task of managing the project to the agent who has an expertise in project management. When delegating, the principal offers a contract with a compensation structure $(f, b)$, where $f$ is a fixed transfer paid to the agent in $t=0$ upfront and $b$ is a bonus paid in the state $\tilde{R}=R$ in either $t=1$ or $t=2$. If the agent declines the principal's offer, she enjoys an outside option which gives $\bar{u}>0$.

[Figure 1] The project return structure

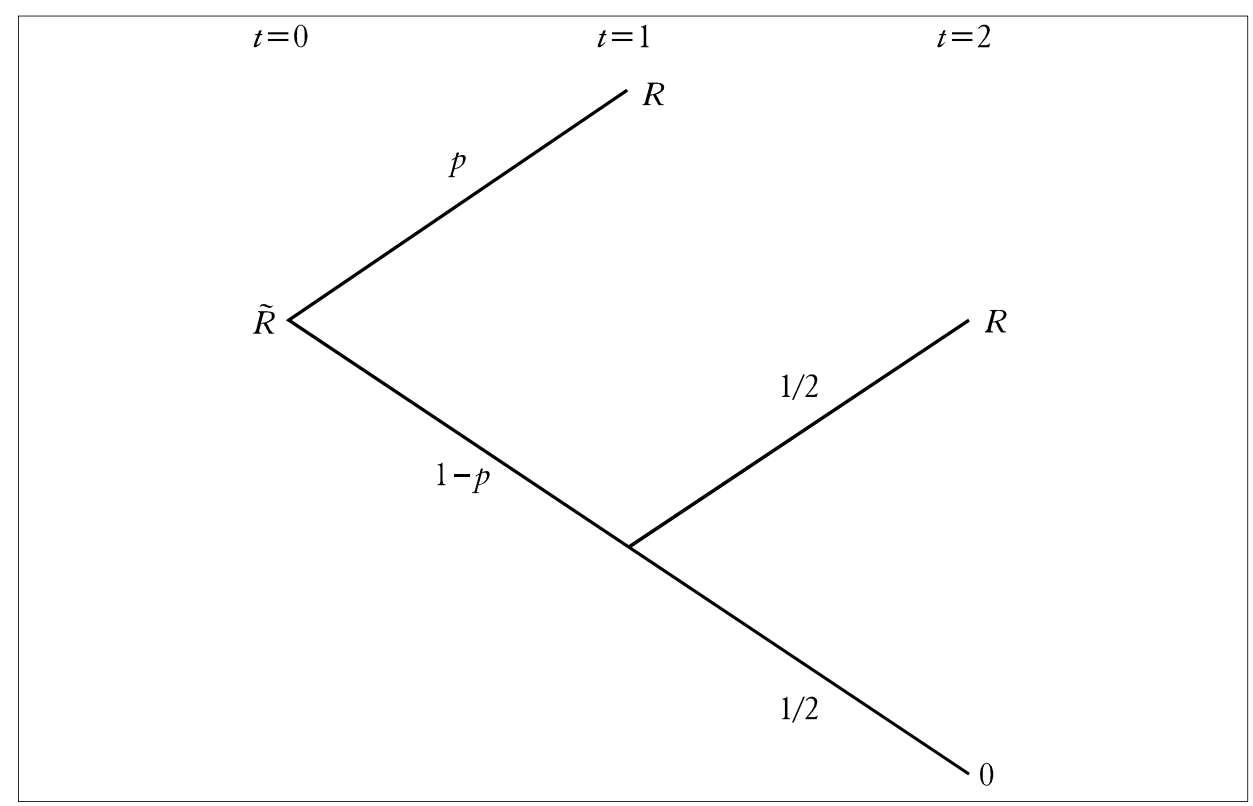

After accepting the principal's offer, the agent decides whether or not to put an effort on the project in $t=0$. If the agent makes an effort, the probability of $\tilde{R}=R$ in $t=1$ will be increased by $\Delta>0$, but the agent suffers from a cost $c>0 .{ }^{5} \mathrm{We}$ assume that the principal cannot observe whether or not the agent effortfully manages the project, which gives rise to a standard moral hazard problem à la Holmstrom (1979).

${ }^{4}$ The return structure of the project, or uncertainty about the project, is similar to Bolton et al. (2011).

${ }^{5}$ We imported this framework of moral hazard from Holmström and Tirole (2011). 
There is an additional source of incentive misalignment in information transmission in $t=1$. Specifically, if the project does not give return in $t=1$, we assume that the agent receives additional information about the prospect of the project. Specifically, we assume that the agent privately receives a noisy but informative signal $s \in\{0, R\}$, where

$$
\operatorname{Pr}(s=R \mid \tilde{R}=R)=\operatorname{Pr}(s=0 \mid \tilde{R}=0)=\sigma \in\left(\frac{1}{2}, 1\right)
$$

After observing the signal, the agent reports a message $m=\{0, R\}$ about the observed signal $s \in\{0, R\}$ to the principal. However, we assume that the agent's message $m$ is unverifiable, which creates an incentive misalignment problem in information transmission à la Crawford and Sobel (1982). To incorporate the social and regulatory cost of misrepresenting the information, we further assume that the agent suffers from a cost $L>0$ if she misreports private information (i.e., $m \neq \mathrm{s}$ ) to the principal (Kartik, 2009).

[Figure 2] The principal's payoff structure

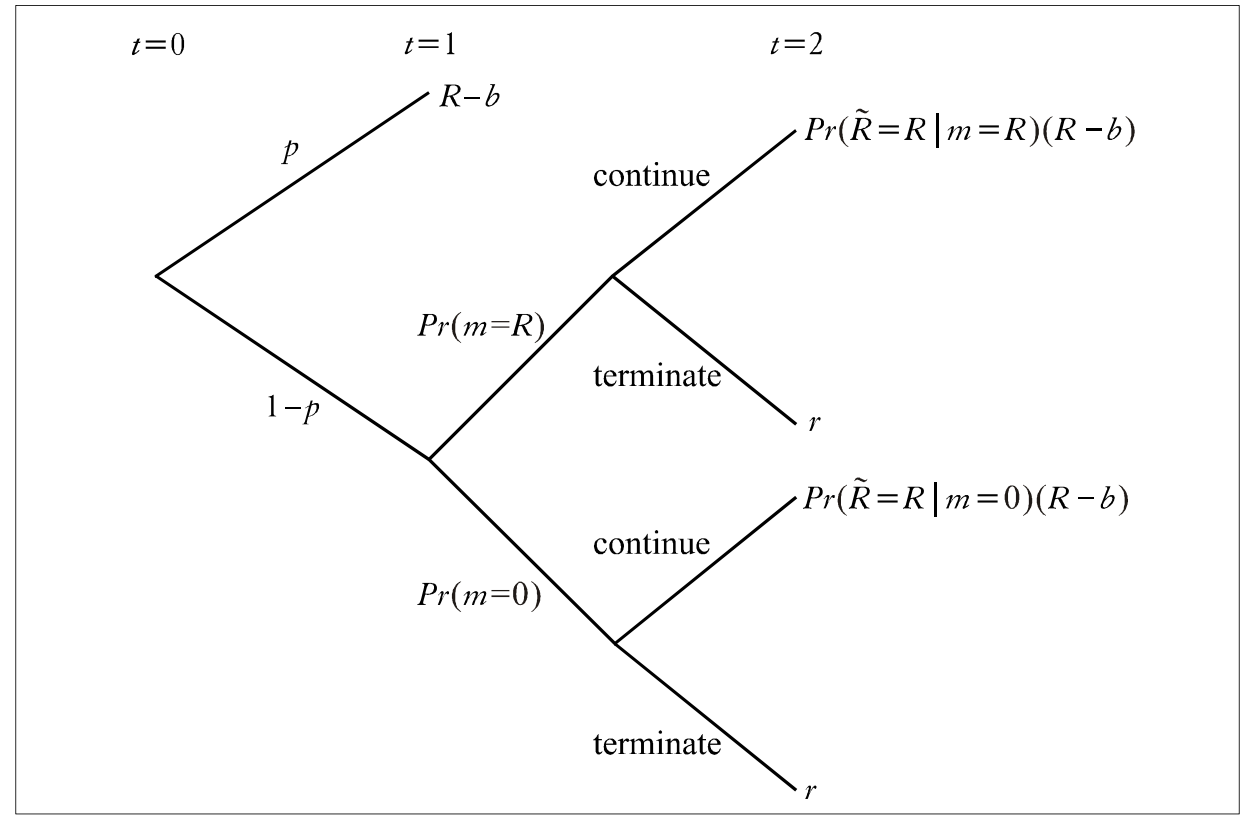

After receiving the message, the principal decides whether or not to terminate the contract with the agent. If the contract is terminated in $t=1$, the principal gets a payoff $r \in(0, R)$ while the agents gets nothing. We assume that if the principal perfectly knew which of signals $s \in\{0, R\}$, he would continue the contract if and 
only if the project is promising (i.e., $s=R$ ). More specifically, we assume

$$
\sigma R>r>(1-\sigma) R
$$

Figure 2 illustrates the payoff structure of the principal at every date.

The timeline of actions in this game is following:

1. In $t=0$, the principal offers a contract $(f, b)$ of delegating the management of the project, and the agent decides whether or not to accept the offer.

2. If the agent accepts the principal's offer, she decides whether or not to take an effort.

3. If the project does not give any return in $t=1$, the signal $s$ is realized and the agent sends a message $m$ to the principal.

4. After receiving the message, the principal decides whether or not to continue the contract with the agent.

5. When the project yields a return in either $t=1$ or $t=2$, the return is distributed to the players according to the contract.

\subsection{Interpretation}

In this section, we discuss how our model captures important features of contractual relationship between economic agents that involves both ex-ante moral hazard and ex-post strategic information transmission problems. In fact, our model is well suited to private equity funds (henceforth PEF) consisting of limited partners (henceforth LPs) which provide money to the PEF, and a general partner (henceforth GP) which provide expertise in managing the PEF.

To manage PEFs funded by LPs, GPs are required to have expertise on several fronts. First, GPs must be superior in searching and purchasing non-traditional financial assets worth investing. PEFs typically invest in so-called "alternative investment assets," such as startup companies, distressed firms under restructuring, real estates, and infrastructure projects. However, there is no public exchange since there are only a small group of investors infrequently trading these types of asset. Thus, investors, although having a strong interest in alternative investment assets, face difficulty in finding prospective sellers. The absence of a public exchange also makes the investors to value alternative investment assets because there are no market prices for these assets. In the same context, PEFs face difficulty in finding a wealthy buyer although the investors decide to realize the return from their investment: alternative investment assets (such as commercial real estate properties) are usually traded at a very expensive price per unit. Furthermore, specialized managerial skills are required to enhance the value of alternative investment assets: the investments cannot be profitable if the PEFs fail to successfully restructure 
distressed firms. To overcome these difficulties, LPs need GPs specialized in collecting information on potential investment opportunities, business networks with prospective buyers and sellers of the assets, and experiences of managing alternative investment assets.

However, GPs' expertise in alternative investments ironically creates incentive misalignment problems within PEFs. First, GPs may not put their full effort on their asset management tasks unless their efforts are sufficiently rewarded. Since GPs' efforts are indirectly judged by their performance - the return of the investments, LPs face a typical moral hazard problem. Second, GPs may misuse their superiority in collecting information on alternative investment assets to their own favors. Suppose a GP, in the middle of managing a PEF, privately observes that their assets will not be sold at high prices in the end. ${ }^{6}$ However, the GP may not want to truthfully report such a negative prospect of their investment to LPs. On the first hand, LPs possibly request early liquidation of investments due to the concern about future losses. Under this circumstance, the GP cannot receive a performance-based payment from managing the PEF. Furthermore, the early liquidation also harms the GP's reputation: they will be perceived as those with poor fund management skills. For this reason, the GP has an incentive to strategically misrepresent the valuation report to the LPs.

Roger (2013) focused on the same issue, but there are a number of features of PEFs (and other economic institutions) not incorporated by Roger (2013). First, Roger (2013) assumes that agents receive their payments based on reported performances. However, due to the difficulty of valuing alternative investment assets, PEFs do not pay a bonus to GPs based on the mid-term report. Rather, many of PEFs adopt the following fee structure: GPs receive performance-based payments contingent on the realized return (which is typically $20 \%$ of the total net return from the investment); GPs receive annual upfront "management fees" from LPs to cover the administrative costs of managing PEFs (mostly 2\% of collected money), which are unconditional on the performance of the funds (Metrick and Yasuda, 2010). Second, as was presented in the introduction, GPs may suffer from explicit and implicit penalties if they are found to misrepresent valuation results of the invested assets to LPs.

To understand the incentive structures that involve GPs and LPs in PEFs more clearly, we need to develop an alternative well-suited model. In this context, we add new elements to our model that facilitate to analyze the aforementioned dual incentive problems that PEFs are involved. First, we consider fee structures with fixed transfers and a performance-based payments as effective incentive scheme to

\footnotetext{
${ }^{6}$ More precisely, it is a third-party auditor who reviews the valuation results before being distributed to the LP. However, the auditor, mostly accounting agencies, are paid by the GP for the auditing task, making the auditor susceptible for colluding with the GP (Celik, 2009).
} 
resolve both moral hazard and strategic communication problems. Particularly, we will discuss why the sensitivity of the remuneration schemes to the final outcome is important to tackle this problem. Second, our paper incorporates the cost of lying into the model in order to capture the costs of misreporting the value of the invested assets. In this setup, we can effectively analyze how the degree of regulatory enforcements on auditing - represented by the cost of lying - influence the optimal contract design.

There are also several additional assumptions made for the stylization of our model. First, we assume that the agent receives the same bonus payment $b$ whether the project gives a positive return in $t=1$ or $t=2$. This assumption is made to incorporate a real-world feature of PEFs into our model. Specifically, as mentioned by Metrick and Yasuda (2010), it is a customary practice in PEFs that bonus schemes for GPs are not renegotiated although the returns of investments are realized either earlier or later than the scheduled maturity date of PEFs. ${ }^{7}$ With this stylization, we can provide positive analysis of how the customary fee structure widely accepted by PEFs can incentivize GPs to duly manage the investments. Furthermore, we assume that the agent's decision on making an effort increases the probability of high return in $t=1$ only. Such an assumption is made to simplify our analysis by separating the timing of the arrival of the moral hazard problem from that of the strategic communication problem. However, our main result is not qualitatively changed even if we generalize this setup by assuming that the agent's action can influence the probability of high return both periods.

Second, we assume that the agent will get a positive payoff when she rejects the principal's offer, while she gets zero payoff if she fails to yield high return from the project. This can be viewed that the agent willingly takes on an inherent risk, such as a negative reputational consequence, of failing the project when she accepts the principal's offer. In this context, the positive payoff from the outside option can be interpreted as the agent's lifetime expected utility from avoiding the risk of poorly managing the principal's project.

Lastly, we assume that all players in our model do not discount their payoffs later in $t=2$ than those realized at earlier dates. If the players heavily discounts date- 2 payoffs relative to the payoffs realized in $t=0$ or 1 , they will not take into account the ex-post incentive problem in communication as much as moral hazard. Since our main research focus is the dual incentive problem, it will more appropriate to postulate that neither the principal or the agent neglects a consequence of the date-1 communication. Note also that our main result is qualitatively unchanged unless all players in the model heavily discount their date- 2 payoffs relative to their date-1

\footnotetext{
${ }^{7}$ A number of institutional investors and PEF managers in Korea were interviewed to confirm this customary fee structure practiced in PEFs. The author acknowledges that the interviewees wanted their identities to be kept anonymous due to their concern with conflicting interests.
} 
payoffs.

\section{Equilibrium Analysis}

In this section, we study the strategic interplay between the principal and the agent in $t=0,1$. Specifically, we first characterize a partial equilibrium in $t=1$ for a given contract $(f, b)$, in which the agent reports her private information and the principal decides whether or not to continue the contract. In particular, we focus on how the bonus payment $b$ influences strategic decisions made by both players ex post. By applying this analytical result, we sequentially solve the ex-ante optimal contract design problem facing the principal in $t=0$.

\subsection{Partial Equilibrium in $t=1$ à la Kartik (2009)}

We characterize a Perfect Bayesian equilibrium (henceforth called an "equilibrium") at date 1 . Specifically, we focus on a partially pooling equilibrium in which (i) the agent, after observing $s=0$, sends the message $m=R$ to the principal with probability $\lambda \in[0,1]$, but truthfully reports after observing $s=R$; (ii) the principal continues the contract with probability $\gamma \in[0,1]$ after receiving $m=R$, but always terminates the contract after receiving $m=0$. The only other possible equilibrium outcome is that the agent always sends $m=0$ whether she observes $s=0$ or $s=R$. However, as we prove in Appendix A, such an outcome does not satisfy D1 refinement (Cho and Sobel, 1990). ${ }^{8}$

Fix an arbitrary contract $(f, b)$, and suppose the agent receives $s=0$ in $t=1$. Given the principal's strategy $\gamma \in[0,1]$, the agent gets the expected payoff $\gamma(1-\sigma) b-L$ if she reports $m=R$. Since the agent gets zero payoff if she truthfully reports her signal, the agent with $s=0$ has an incentive to lie if and only if

$$
\gamma(1-\sigma) b-L \geq 0
$$

After receiving $s=0$, the agent's incentive to lie is closely related to the bonus payment $b$ she receives upon the realization of high return in $t=2$. If $b$ is too low, the agent will truthfully report her information to avoid the cost of lying. From (3), we can derive a latent variable $\hat{\gamma}$ which makes the agent with $s=0$ indifferent between reporting truthfully and lying:

\footnotetext{
${ }^{8}$ It is more plausible that the agent has an incentive to exaggerate the prospect of the delegated project at the expense of the lying cost, not the other way around.
} 


$$
\hat{\gamma}:=\frac{L}{(1-\sigma) b} .
$$

One can easily find that $\hat{\gamma}$ is decreasing in $b$. That is, the principal must continue the contract with a smaller probability if the agent has a stronger incentive to misreport her information due to the increased bonus payment.

We next turn to the principal's equilibrium strategy corresponding to the agent's strategy $\lambda$ and the bonus payment $b$. After observing $m=R$, the principal updates his belief about the project as

$$
\begin{aligned}
\operatorname{Pr}(s=R \mid m=R)= & \sigma \frac{\operatorname{Pr}(s=R)}{\operatorname{Pr}(s=R)+\lambda \operatorname{Pr}(s=0)} \\
& +(1-\sigma) \frac{\lambda \operatorname{Pr}(s=0)}{\operatorname{Pr}(s=R)+\lambda \operatorname{Pr}(s=0)}=\frac{\sigma+\lambda(1-\sigma)}{1+\lambda} .
\end{aligned}
$$

Note that $\operatorname{Pr}(s=R \mid m=R)$ is decreasing in $\lambda$. Intuitively, the principal becomes more optimistic after $m=R$ as the agent with $s=0$ is less likely to lie to the principal. The principal's net expected payoff from continuing the contract after $m=R$ is thus equal to

$$
\operatorname{Pr}(s=R \mid m=R)(R-b)-r=\frac{\sigma+\lambda(1-\sigma)}{1+\lambda}(R-b)-r
$$

Intuitively, the principal becomes more willing to continue the contract when the agent with $s=0$ is less likely to lie. Furthermore, the principal's incentive to continue the contract after $m=R$ decreases with $b$ : a bigger bonus means the principal must yield a large share of the project return with the agent.

From (6), one can find that the principal continues the contract if and only if

$$
\frac{\sigma+\lambda(1-\sigma)}{1+\lambda}(R-b)-r \geq 0
$$

Just like $\hat{\gamma}$, we can find another latent variable $\hat{\lambda}$ that makes the principal indifferent between continuing the contract and terminating it:

$$
\hat{\lambda}:=\frac{\sigma-\frac{r}{R-b}}{\frac{r}{R-b}-(1-\sigma)} .
$$


Note that $\hat{\lambda}$ is decreasing in $b$. Indeed, the principal becomes unwilling to continue the contract when he has to yield a large share of the project return to the agent. Hence, it is necessary for the agent to be more likely to truthfully report her information so that the principal, after receiving $m=R$, is convinced that continuing the contract gives a higher expected payoff than terminating it .

Let $\left(\gamma^{*}, \lambda^{*}\right)$ denote the equilibrium strategies of two respective players in $t=1$. Applying the results above, we can establish the equilibrium as follows.

Theorem 1 (Equilibrium at date 1). For each $b$, there exists a unique date-1 partial equilibrium, characterized by two cutoffs $\underline{b}^{*} \leq \bar{b}^{*}$.

(i) If $b \leq \underline{b}^{*}$, the agent with $s=0$ never lies $\left(\lambda^{*}=0\right)$ and the principal surely continues the contract $\left(\gamma^{*}=1\right)$ after $m=R^{\text {; }}$

(ii) If $b \in\left(\underline{b}^{*}, \bar{b}^{*}\right]$ the agent with $s=0$ always lies $\left(\lambda^{*}=1\right)$ and the principal surely continues the contract $\left(\gamma^{*}=1\right)$ after $m=R$;

(iii) If $b>\bar{b}^{*}$, the agent with $s=0$ lies with probability $\lambda^{*}=\min \{\hat{\lambda}, 0\}$ and the principal continues the contract with probability $\gamma^{*}=\hat{\gamma}$ after $m=R$.

Figure 3 graphically illustrates how the date-1 partial equilibrium strategies are pinned down by the bonus payment $b$. Suppose first $b$ is sufficiently low, i.e., $b \leq \underline{b}^{*}$. Then, the agent with $s=0$ no longer has an incentive to exaggerate the expected value of the project since lying is too costly (i.e., $(1-\sigma) b-L \leq 0)$. Since the agent is believed to truthfully report her information, the principal also fully trust the agent's report, and therefore, continues the contract if and only if he receives good news from the agent (i.e., $m=R$ ).

Next, suppose $b \in\left(\underline{b}^{*}, \bar{b}^{*}\right]$. Although $b$ becomes relatively high, it is not high enough for the principal to perceive that it is very costly to continue the contract. Hence, the principal approves of continuing the contract even if the agent's report is totally uninformative. By contrast, $b$ is sufficiently high for the agent with $s=0$ so that she, although the odds are low, bets for a big bonus from high performance in the future. Thus, the agent's optimal strategy after observing $s=0$ is to surely lie to the principal-i.e., $m=R$ with probability 1 .

Lastly, suppose $b>\bar{b}^{*}$. We first check that there is no pure-strategy equilibrium. Suppose the agent with $s=0$ always misreports to the principal. This means that $m=R$ conveys no additional information, so the principal holds the belief same as the prior after receiving $m=R$. However, the principal will not continue the contract after $m=R$ because he is not convinced that the project is likely to yield a high net return. Knowing that the principal will surely terminate the contract after $m=R$, the agent with $s=0$ would rather truthfully report her information to avoid the lying cost $L$. Next, suppose the agent with $s=0$ truthfully reports her information. Then, from (2), the principal believes the agent sends $m=R$ if and only if $s=R$, and thus will surely continue the contract after $m=R$. Since 
$(1-\sigma) b-L>0$, however, the agent with $s=0$ will surely deviate and misreport $m=R$. Thus, the only possible equilibrium is the mixed-strategy equilibrium in which the principal, after receiving $m=R$, randomizes in continuing the contract to make the agent indifferent between truthfully reporting and lying, and vice versa. ${ }^{9}$

One noteworthy feature is that the bonus scheme crucially determines the incentive structure of the agent to share her private information with the principal. First, any contract with a low bonus payment (i.e., $b \leq \underline{b}^{*}$ ) completely resolves the strategic communication problem. Second, for all contracts with a high bonus payments $b>\underline{b}^{*}$, an increase in $b$ has mixed effects on the information transmission. On the first hand, a higher $b$ decreases the probability that the agent misreports her private information, making the message $m=R$ more accurate. On the other hand, an increase in $b$ also reduces the probability that the principal continues the contract after receiving $m=R$. Hence, an increase in $b$ worsens the ex-post incentive misalignment in communication: although the agent sends more informative message, the principal is more likely to make a "wrong" decision. In sum, the probability of lying exhibits non-monotonicity with respect to the level of the performance-based payment, which is distinct from Roger (2013).

[Figure 3] The date-1 partial equilibrium

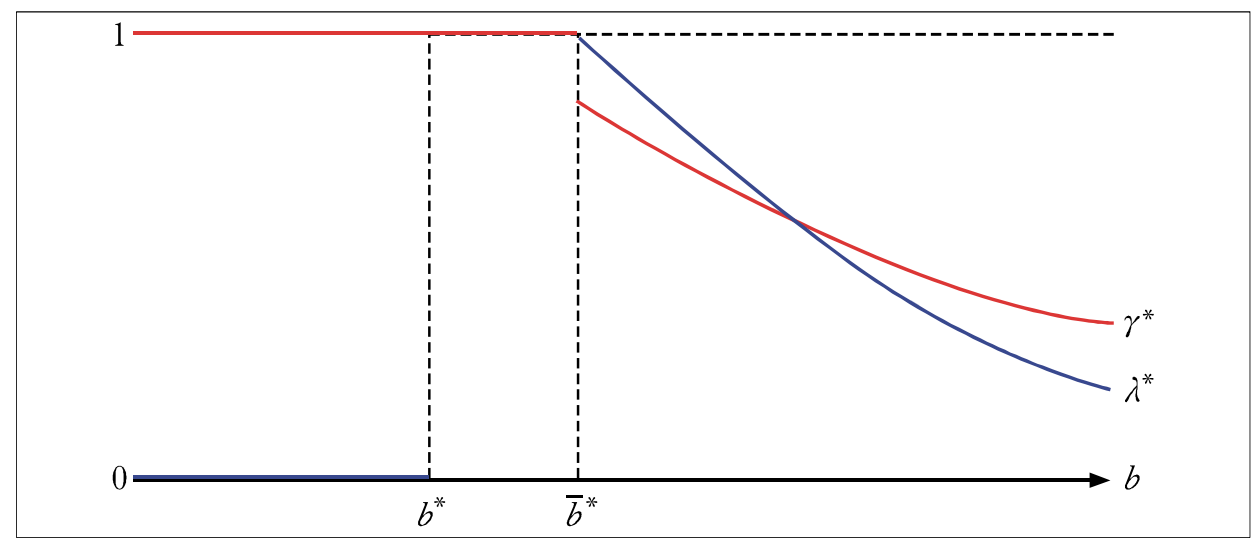

Another interesting implication is that the principal will face a dilemma when

\footnotetext{
${ }^{9}$ A relevant real-world example is the Abraaj case. In 2017, institutional investors in Abraaj's \$1 billion healthcare fund, including the Gates Foundation and World Bank, grew suspicious about why their money was not being spent for scheduled investments. To restore investors' confidence, Abraaj Group hired KPMG to audit the fund and released the result that the money was not misappropriated. However, the investors did not give credit to this report and conducted an independent forensic audit to find that Abraaj Group misused investors' money to pay for operational expenses of the other funds. Go to https:/www.ft.com/content/31ab6f82-a79a-11e8-926a-7342fe5e173f and https: //www.ft.com/ content/c31e6040-78b0-11e9-b0ec-7dff87b9a4a2 for more details.
} 
solving the optimal contract design problem ex ante, which echoes with Roger (2013). To provide the agent with an incentive to put a full effort, the principal needs to design a compensation scheme to be highly contingent on the outcome of the project. On the flip side, a big bonus may distort the agent's incentive of transmitting her private information: the agent may want to exaggerate the prospect of the project to induce the principal to continue the contract inappropriately. To address this ex-post communication inefficiency, the principal may need to offer a low bonus payment to reduce the gains from exaggerating the information. However, such a pay structure possibly fails to address the ex-ante moral hazard problem, lowering the overall expected surplus from the project. Alternatively, if the moral hazard problem requires the bonus payment to be far higher than $\underline{b}^{*}$, the principal would rather offer exceptionally big bonus payment to induce the agent to transmit her private information accurately. We further study how the principal can find an optimal contract that solves the dual incentive problem ex ante.

\subsection{Optimal Contract Design at Date 0}

In this section, we find an optimal contract $(f, b)$ that maximizes the principal's total payoff at date 0 . As was discussed in the previous section, there is a tradeoff of the bonus payment: a low bonus payment may be necessary to provide the agent with an incentive not to misrepresent her privation information; a low bonus payment discourages the agent from making an effort on the delegated task. We analyze how an optimal contract is determined by the cost of lying $L$, which influences the agent's incentive to strategically misreport her private information $e x$ post.

To facilitate the analysis, we first introduce the regularity conditions:

Assumption 1. (i) $\frac{1}{2}(R-r) \geq \frac{c}{\Delta} ;$ (ii) $\underline{b}^{*}=\bar{b}^{*}$; (iii) $(p+\Delta) R+(1-(p+\Delta))\left(\frac{1}{2} R+\right.$ $\left.\frac{1}{2} r\right)-\bar{u}-c>0 ;(i v) \bar{u}-(1+p) \frac{c}{\Delta}>0$.

Assumption 1-(i) is equivalent to

$$
(p+\Delta) R+(1-(p+\Delta))\left(\frac{1}{2} R+\frac{1}{2} r\right)-c \geq p R+(1-p)\left(\frac{1}{2} R+\frac{1}{2} r\right)
$$

This inequality implies that that the principal must prioritize addressing the exante moral hazard problem in order to maximize his utility. In this context, we throughout restrict our attention to the contracts that incentivize the agent to make an effort in $t=0$. Assumption 1-(ii) rules out the possibility that there does not exist any $(f, b)$ that supports a date-1 partial equilibrium with $\lambda^{*}=1$ and 
$\gamma^{*}=0$. This assumption is made to highlight the main result clearly: the main result of this paper is qualitatively unchanged even if we relax this assumption. ${ }^{10}$ Lastly, Assumption 1-(iii) is a necessary condition for the existence of an optimal contract, and Assumption 1-(iv) ensures that an optimal contract must be generous on average enough to attract the agent. We throughout abbreviate the date-1 probability of $\tilde{R}=R$ to $\tilde{p} \in\{p, p+\Delta\}$ and the cost of the effort to $\tilde{c} \in\{0, c\}$, respectively, that vary with the agent's decision on making an effort in $t=0$.

When solving the optimal contract design problem, the principal has to take into account the ex-post consequence of the bonus payment $b$ on the agent's ex-post incentive to misrepresent her private information in $t=1$. By Theorem 1 , there are two possible outcomes: the agent never misreports $\left(\lambda^{*}=0\right)$ after receiving $s=0$; the agent randomizes in misreporting with probability $\lambda^{*} \in(0,1]$ and the principal randomizes in continuing the contract with probability $\gamma^{*} \in[0,1]$. In this context, we find an optimal contract in the following steps: $(i)$ we first find an optimal contract of all contracts that yield the date- 1 partial equilibrium with $\lambda^{*}=0$; (ii) we next find an optimal contract of all contracts that yield the date-1 partial equilibrium with $\lambda^{*} \in(0,1]$; (iii) we lastly find an optimal contract by checking which of these two contracts yields a higher total expected payoff to the principal than the other.

First, Consider the contracts $(f, b)$ that induces the date-1 equilibrium with $\lambda^{*}=0$. Since the agent truthfully reports her private information in $t=1$, her total net expected payoff from accepting the principal's offer $(f, b)$ is

$$
f+\tilde{p} b+(1-\tilde{p}) \frac{1}{2} b-\tilde{c}-\bar{u}
$$

Furthermore, the outcome generated by $(f, b)$ must be consistent with the conjectured equilibrium. Since the agent makes an effort on the project in equilibrium, we must have the following incentive compatibility constraint:

$$
f+(p+\Delta) b+(1-(p+\Delta)) \frac{1}{2} b-c-\bar{u} \geq f+p b+(1-p) \frac{1}{2} b-\bar{u},
$$

Which is rewritten as

\footnotetext{
${ }^{10}$ More precisely, either the equilibrium with $\lambda^{*} \in(0,1)$ or the equilibrium with $\lambda^{*}=1$, but not both of them, exists for every value of $L$. We simplify our analysis because the main objective of the analysis is to investigate whether there exists an optimal contract that fully resolves both moral hazard and inefficient communication problems, not to establish all possible equilibrium outcomes.
} 


$$
b \geq b^{*}=\frac{2 c}{\Delta} .
$$

Note that $b^{*}$ - the lower bound of the bonus payment that resolves the ex-ante moral hazard problem - does not vary with the lying cost $L$. Namely, the principal does not have to compensate the agent for the ex-post cost of lying since the agent is expected to report her private information truthfully in $t=1$.

Moreover, the bonus payment $b$ must not be too high to incentivize the agent to lie in $t=1-$ i.e., $(1-\sigma) b-L<0$. Hence, by applying (10), we have

$$
(1-\sigma) b-L<0 \Rightarrow L>(1-\sigma) b \geq L^{*}:=\frac{2 c(1-\sigma)}{1+\Delta}
$$

That is, the cost of lying must be sufficiently high for the agent to refrain from misreporting her own information. Note that $L^{*}$ - the lower bound of $L$ 's that induce the agent not to lie - is decreasing in $b:$ a lower bonus payment provides the agent with an incentive to report truthfully. In an extreme case, a low cost of lying $L=L^{*}$ is enough to yield no misreporting by the agent in $t=1$ if the principal offers the lowest bonus payment $b^{*}$ that resolves the ex-ante moral hazard problem.

Lastly, the agent must prefer accepting the principal's offer to her outside option, leading to the following participation constraint:

$$
f+(p+\Delta) b+(1-(p+\Delta)) \frac{1}{2} b-c-\bar{u} \geq 0
$$

The optimal contract must solve the principal's problem of minimizing the expected compensation to the agent. Hence, the principal must solve the following cost minimization problem:

$$
\min _{(f, c)} f+(p+\Delta) b+(1-(p+\Delta)) \frac{1}{2} b=\min _{(f, c)} f+\frac{1}{2}(1-(p+\Delta)) b
$$

subject to $(10)-(12)$. One of the contracts that solves the above problem is $\left(f^{*}, b^{*}\right)$, where

$$
f^{*}=\bar{u}-(1+p) \frac{c}{\Delta}, \quad b^{*}=\frac{2 c}{\Delta}
$$

Thus, we have the following observation. 
Theorem 2. The equilibrium with $\lambda^{*}=0$ exists if and only if $L>L^{*}$. Out of all contracts inducing the equilibrium with $\lambda^{*}=0$, a contract $\left(f^{*}, b^{*}\right)$ maximizes the principal's total expected payoff.

One interesting feature is that the cost of lying $L$ must be necessarily high. If $L$ is low, the agent becomes strongly tempted to misreport her private signal. Since the principal does not want to be ill-informed by the agent, he may offer a contract with a small bonus payment to provide the agent with an incentive not to lie ex post. Since $L$ is low, however, such a $b$ cannot address the ex-ante moral hazard problem.

It is also worth noting that $\left(f^{*}, b^{*}\right)$ is not a unique contract offer that maximizes the principal's expected payoffs. Indeed, the linearity of the cost minimization problem in (13) implies that there are infinitely many other optimal contracts. One important feature of $\left(f^{*}, b^{*}\right)$ is that the fee structure yields the lowest implementable bonus payment that resolves both ex-ante moral hazard and ex-post inefficient information transmission problems.

Consider next the contracts that yield the date- 1 mixed-strategy equilibrium with $\lambda^{*} \in(0,1]$ and $\gamma^{*} \in[0,1]$. By Theorem 1 , the agent's total expected payoff over two periods given a contract $(f, b)$ is

$$
\begin{aligned}
& f+\tilde{p} b+(1-\tilde{p})\left[\operatorname{Pr}(s=0) \lambda^{*}\left\{\gamma^{*}(1-\sigma) b-L\right\}+\operatorname{Pr}(s=R) \gamma^{*} \sigma b\right]-\tilde{c}-\bar{u} \\
& =f+\tilde{p} b+(1-\tilde{p}) \frac{1}{2}\left[\lambda^{*}\left\{\gamma^{*}(1-\sigma) b-L\right\}+\gamma^{*} \sigma b\right]-\tilde{c}-\bar{u} .
\end{aligned}
$$

Since $\gamma^{*}(1-\sigma) b-L=0$ in equilibrium, (15) is rewritten as

$$
f+\tilde{p} b+(1-\tilde{p}) \frac{\sigma}{2(1-\sigma)} L-\tilde{c}-\bar{u}
$$

There are also necessary conditions for the outcome generated by a contract $(f, b)$ to be consistent with the conjectured equilibrium. Just as before, $(f, b)$ must make the agent prefer making an effort in $t=0$. Since the agent is indifferent between misreporting and truthfully reporting in $t=1$, we have the following condition:

$$
f+(p+\Delta) b+(1-(p+\Delta)) \frac{\sigma}{2(1-\sigma)} L-c-\bar{u} \geq f+p b+(1-p) \frac{\sigma}{2(1-\sigma)} L-\bar{u}
$$

which is equivalent to 


$$
b \geq \frac{c}{\Delta}+\frac{\sigma}{2(1-\sigma)} L .
$$

Furthermore, the condition (3) implies that the bonus payment $b$ must be sufficiently high so that $(1-\sigma) b-L>0$. Lastly, a contract $(f, b)$ must satisfy the participation constraint:

$$
f+\tilde{p} b+(1-\tilde{p}) \frac{1}{2}\left[\lambda^{*}\left\{\gamma^{*}(1-\sigma) b-L\right\}+\gamma^{*} \sigma b\right]-\tilde{c}-\bar{u} \geq 0
$$

The principal can maximize his expected payoff by minimizing the expected transfer to the agent. To this end, the condition (17) must be binding. Furthermore, $b$ must be high enough to satisfy (3).

Therefore, the principal's cost minimization problem is

$$
\min _{f, b} f+(p+\Delta) b+(1-(p+\Delta)) \frac{1}{2}\left(\sigma+(1-\sigma) \lambda^{*}\right) \gamma^{*} b
$$

Since (17) must be binding and $(1-\sigma) \gamma^{*} b-L=0$ in equilibrium, the principal's optimization problem above is simplified as

$$
\min _{b \geq \frac{c}{\Delta}+\frac{\sigma}{2(1-\sigma)} L} \frac{1}{2}(1-(P+\Delta)) \lambda^{*} L
$$

Since $\lambda^{*}$ is decreasing in $b$, the principal can minimize the total cost by increasing $b$ as much as possible. Therefore, the optimal contract, denoted by $\left(f^{* *}, b^{* *}\right)$, is

$$
f^{* *}=0, \quad b^{* *}=\left(\frac{1}{p+\Delta}\left[\bar{u}+c-(1-(p+\Delta)) \frac{\sigma}{2(1-\sigma)} L\right]\right)
$$

if $b^{* *}$ satisfies the conditions $(1-\sigma) b^{* *}-L>0$ and (16). Since $(1-\sigma) b^{* *}-L$ is decreasing in $L$, we have the following observation.

Theorem 3. There exists a $L^{* *}$ such that $\left(f^{* *}, b^{* *}\right)$ of all contracts inducing the equilibrium with $\lambda^{*}>0$ maximizes the principal's total expected payoff for every $L<L^{* *}$.

Note from (19) that the optimal contract $\left(f^{* *}, b^{* *}\right)$ offers the highest bonus 
payment satisfying both (16) and (17). The condition (18) indeed implies that the principal can increase the expected surplus from the delegated project by lowering the probability of misreporting ex post. This can be achieved by raising the bonus payment so that the principal will not continue the contract after $m=R$ unless the agent reports truthfully with a high probability. This implies that the principal can maximize his total expected payoff by providing the agent with a strong incentive to truthfully report her private information ex post.

From Theorem 2 and 3, we can find an optimal contract.

Theorem 4. If $L<L^{*}$, then $\left(f^{* *}, b^{* *}\right)$ maximizes the principal's total expected payoff; if $L \geq L^{*}$, then $\left(f^{*}, b^{*}\right)$ is the optimal contract.

[Figure 4] The optimal contract

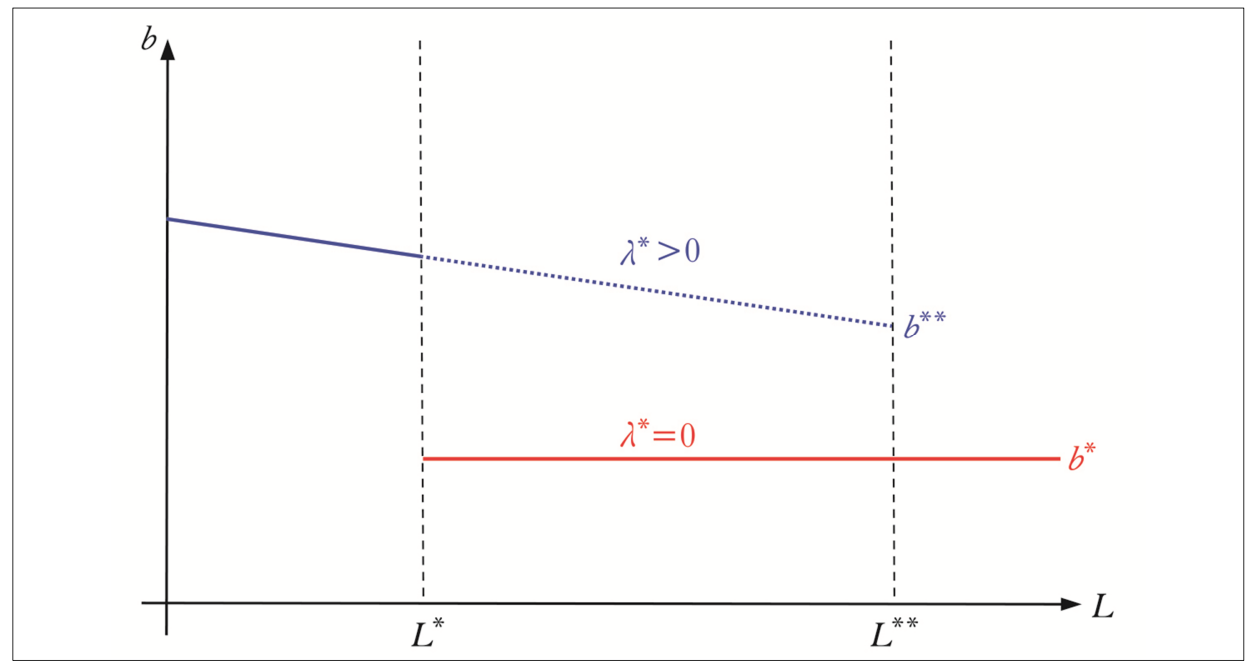

As depicted by Figure 4, the optimal contract is pinned down by the cost of lying $L$. If $L<L^{*}$, only the equilibrium with $\lambda^{*}>0$ is possible, so the contract $\left(f^{* *}, b^{* *}\right)$ maximizes the principal's expected payoff. If $L \in\left[L^{*}, L^{* *}\right)$, both types of equilibria are implementable. However, the principal suffers from losses incurred by the agent's lying only in the equilibrium with $\lambda^{*}>0$ : the principal wrongly continues the contract with probability $\lambda^{*} \gamma^{*} \operatorname{Pr}(s=0)$; he wrongly terminate the contract with probability $\operatorname{Pr}(s=R)\left(1-\gamma^{*}\right)$. Since the ex-ante moral hazard problem is resolved in both equilibria, the principal prefers inducing the equilibrium with $\lambda^{*}=0$. Furthermore, one can easily find $b^{*}<b^{* *}$ for any $L \in\left[L^{*}, L^{* *}\right)$, which implies that the contract offering a low bonus payment can give the highest surplus from the project to the principal.

The adverse effect of the bonus payment is even more pronounced if the 
principal is allowed to adjust bonus schemes to the timing of the realization of the return. Suppose the principal can offer a contract with fee structure $\left(f, b_{1}, b_{2}\right)$, where $b_{1}$ and $b_{2}$ are time-dependent bonus payments when the project yields high return at $t=1$ and $t=2$, respectively. With such a fee structure, the principal can fully eliminate the agent's incentive to lie by making the date- 2 bonus pay $b_{2}$ sufficiently low (or even $b_{2}=0$ ) and the date-1 payoff sufficiently high to resolve the ex-ante moral hazard problem and encourage the agent to accept the principal's offer. This result suggests that, where possible, the principal should avoid offering bonus schemes that aggravate the inefficient communication problem, but hardly improves the moral hazard problem.

There are several noteworthy features with policy implications for regulating the compensation structure. First, the cost of lying must be necessarily high to obtain the ex-post efficient communication. Due to the dilemma facing the principal, a compensation scheme alone, without the cost of lying, cannot fully address dual incentive misalignment problem that we throughout focus on. Specifically, at a low cost of lying, any bonus scheme to resolve the ex-ante moral hazard problem adversely encourages the agent to misrepresent her private information ex post.

This feature highlights the importance of regulatory enforcement systems as necessary discipline scheme to realign the incentives of fund managers. For example, GPs in PEFs wish LP to hold on their money in the funds until their investments yield high return. Particularly, under lax accounting and auditing standards, GPs can be tempted to overestimate the value of their funds invested in alternative investment assets. Our analytical result suggests that a weak regulatory system may lead to inefficient delay in liquidation of the failing funds.

Second, given a high cost of lying, the principal can resolve the dual incentive misalignment problems by designing the compensation scheme to be loosely tied to the final outcome of the project. This feature sheds light on an important - but relatively unnoticed - role of a fixed upfront fee transferred to the agent regardless of her performance. As was discussed in Section 3.2, GPs in PEFs traditionally collect management fees an annual fixed fee of $2 \%$ of the committed capital from LPs. This fee is supposedly endowed to cover the administrative costs incurred by managing the investments. In a standard moral hazard framework, such a fee structure is inefficient to address the moral hazard problem (Cai et al., 2017). However, our main result suggests that fee structures with weak correlation with the outcome can be an effective incentive scheme. Specifically, such a scheme can provide GPs with an incentive to truthfully report the prospects of the investments, which LPs cannot evaluate due to the lack of expertise. 


\section{Conclusion}

This paper provides analysis of optimal contracts in the presence of ex-ante moral hazard and ex-post strategic communication. Specifically, this paper focuses on the economic activities in which an agent bears a cost of misreporting her privately observed signal about the outcome of her action, which cannot be verified by a principal. In this setup, we find that a fee structure which is relatively insensitive to the stochastic outcome of the agent's action can address inefficiency in the ex-post communication. Our main result sheds light on fixed upfront transfers as an important scheme to address the ex-post incentive problem in communication. 


\section{Appendix}

\section{A Equilibrium Selection with D1 Refinement}

We first show that there cannot exist date-1 equilibrium in which the agent lies (i.e., sends $m=0)$ after observing $s=R$. For exposition, let $\lambda_{R}$ and $\lambda_{0}$ denote the mixed strategies that the agent sends $m=R$ with probability $\lambda_{R}$ after receiving $s=R$ and with probability $\lambda_{0}$ after observing $s=0$, respectively. Moreover, let $\gamma_{R}$ and $\gamma_{0}$ denote the mixed strategies that the principal continues the project with probability $\gamma_{R}$ after receiving $m=R$ and $\gamma_{0}$ after receiving $m=0$, respectively. Suppose there exists an equilibrium with $\lambda_{R}<1$ and $\lambda_{0} \in(0,1]$. Then, the agent, after observing $s=R$, must be indifferent between sending $m=R$ and $m=0$, which implies $\sigma b \gamma_{0}-L \geq \sigma b \gamma_{R}$, and therefore, $\gamma_{0}>\gamma_{R}$. Furthermore, the same indifference condition must be satisfied for the agent with $s=0$, which implies $(1-\sigma) b \gamma_{R}-L \geq(1-\sigma) b \gamma_{0}$. This further implies $\gamma_{R}>\gamma_{0}$, a contradiction. Suppose next there exists an equilibrium with $\lambda_{R} \in(0,1)$ and $\lambda_{0}=0$. Since it is optimal for the principal to continue the project after $m=R$ from (2), we must have $\gamma_{R}=1$. However, the agent with $s=R$ will deviate by sending $m=R$ with probability 1 since $\sigma b>\sigma b \gamma_{0}-L$. Lastly, suppose there is an equilibrium with $\lambda_{R}=1$ and $\lambda_{0}=0$. From (2), it is optimal for the principal to continue the project with probability 1 if $m=0$ and terminate it with probability 1 if $m=R$. However, this implies $(1-\sigma) b \gamma_{R}-L=-L<(1-$ $\sigma) b \gamma_{0}$, hence the agent with $s=0$ will deviate and truthfully report her private signal.

We next show that the equilibrium with $\lambda_{R}=\lambda_{0}=0$ cannot be supported by D1 refinement. Let $\Gamma(s)$ be the set of all $\gamma_{R}$ 's that make the agent with the signal $s \in\{0, R\}$ prefer the deviation strategy $m=R$ to the prescribed equilibrium strategy $m=0$. Then we have

$$
\Gamma(0)=\left\{\gamma_{R} \geq 0:(1-\sigma) b \gamma_{R} \geq(1-\sigma) b \gamma_{0}\right\}=\left\{\gamma_{R} \geq 0: \gamma_{R} \geq \gamma_{0}\right\}
$$

and

$$
\Gamma(S)=\left\{\gamma_{R} \geq 0: \sigma b \gamma_{R} \geq \sigma b \gamma_{0}-L\right\}=\left\{\gamma_{R} \geq 0: \gamma_{R} \geq \gamma_{0}-\frac{L}{\sigma b}\right\}
$$

Hence, we have $\Gamma(0) \subsetneq \Gamma(S)$, and in fact, $\Gamma(S)$ is a unique maximal. Therefore, with D1 refinement, the out-of-equilibrium belief for the agent sending $m=R$ is $\operatorname{Pr}(s=R \mid m=R)=1$. Hence, the agent, regardless of realized signals, will deviate and send $m=R$, which implies the equilibrium with $\lambda_{R}=\lambda_{0}=0$ cannot be 
supported by D1 refinement.

\section{B Proofs}

Proof of Theorem 1. We first define critical values $\tilde{b}_{\gamma}$ and $\tilde{b}_{\lambda}$ such that $\hat{\gamma}\left(\tilde{b}_{\gamma}\right)=1$ and $\hat{\lambda}\left(\tilde{b}_{\gamma}\right)=1$, respectively, and let $\underline{b}^{*}=\tilde{b}_{\gamma} \wedge \tilde{b}_{\lambda}$ and $\bar{b}^{*}=\tilde{b}_{\gamma} \vee \tilde{b}_{\lambda}$. We throughout restrict our attention to the cases $\underline{b}^{*}<\bar{b}^{*}$ under which the proof nests that under the boundary cases $\underline{b}^{*}=\bar{b}^{*}$.

To prove part (i), fix any $b \leq \underline{b}^{*}$. Since $(1-\sigma) b-L \leq 0$, it is optimal for the agent, after observing $s=0$ to send $m=0$ rather than $m=R$, regardless of the principal's strategy. This also implies there is no equilibrium in which the agent with $s=0$ reports $m=R$ with a positive probability. Given $\lambda^{*}=0$, it follows from (2) that it is optimal for the principal to continue the project after $m=R$ and terminate otherwise.

To prove part (ii), fix a $b \in\left(\underline{b}^{*}, \bar{b}^{*}\right]$. For this fixed $b$, the principal prefer continuing the project even after receiving the uninformative message $m=R$, hence $\gamma^{*}=1$. This also implies that any $b \in\left(\underline{b}^{*}, \bar{b}^{*}\right]$ cannot support the equilibrium with $\lambda^{*}>0$ and $\gamma^{*}<1$. Since $(1-\sigma) b-L>0$, the agent strictly prefers sending $m=R$ to the principal after observing $s=0$ given $\gamma^{*}=1$. Thus, we have $\lambda^{*}=1$.

To prove part (iii), fix any $b>\bar{b}^{*}$. We first show that there is no pure-strategy equilibrium. First, suppose a fictitious equilibrium with $\lambda^{*}=1$. Then, the principal strictly prefers terminating the project after receiving $m=R$, this makes the net payoff of the agent with $s=0$ from sending $m=R$ negative. Second, suppose an equilibrium with $\lambda^{*}=0$. Then, from (2) the principal strictly prefers continuing the project after $m=R$ because he rationally infers that only the agent with $s=R$ will send $m=R$. However, this will make the agent with $s=0$ deviate to send $m=R$ since $(1-\sigma) b-L>0$. Therefore, there exists only a mixed-strategy equilibrium for any $b>\bar{b}^{*}$. From (4), the agent with $s=0$ is indifferent between sending $m=R$ and $m=0$ if and only if the principal continues the project with probability $\hat{\gamma}$ after $m=R$. Similarly, from (8), the principal becomes indifferent between continuation and termination of the project after $m=R$ if and only if the agent with $s=0$ sends $m=R$ with probability $\hat{\lambda}$. Therefore, there exists a unique mixed-strategy equilibrium with $\gamma^{*}=\hat{\gamma}$ and $\lambda^{*}=\hat{\lambda}$.

Q. E. D.

Proof of Theorem 2. We first show that for any $L \leq L^{*}$, there is no equilibrium in which the agent never lies $\left(\lambda^{*}=0\right)$ in $t=1$ and manages the project effortfully in $t=0$. In fact, this follows directly from (11) that for any $L \leq L^{*}$, any incentive 
compatible contract $(f, b)$ yields $(1-\sigma) b-L \geq 0$. For any $L \leq L^{*}$, we proved in the main text that the principal can maximize his expected payoff by offering $\left(f^{*}, b^{*}\right)$ defined as (14), which satisfy the incentive compatibility constraint and the participation constraint, and yields the date- 1 equilibrium with $\lambda^{*}=0$.

Q. E. D.

Proof of Theorem 3. We first find $L^{* *}$. From (19) we know $b^{* *}$ is decreasing in $L$. Since $\frac{c}{\Delta}+\frac{\sigma}{2(1-\sigma)} L$ is increasing in $L$, there exists a $\breve{L}$ such that $b^{* *} \geq \frac{c}{\Delta}+$ $\frac{\sigma}{2(1-\sigma)} L$ if and only if $L \leq \breve{L}$. Furthermore, since $\frac{L}{1-\sigma}$ is increasing in $L$, there exists a $\tilde{L}$ such that $(1-\sigma) b^{* *}-L>0$ and (16) if and only if $L<L^{* *}:=$ $\min \{\breve{L}, \tilde{L}\}$. Since the contract $\left(f^{* *}, b^{* *}\right)$ solves the principal's payoff maximization problem by construction.

Q. E. D.

Proof of Theorem 4. Recall from the proof of Theorem 3 that $b^{* *}=\frac{c}{\Delta}+\frac{\sigma}{2(1-\sigma)} L$ at $L=\breve{L}$. Plugging $b^{* *}=\frac{c}{\Delta}+\frac{\sigma}{2(1-\sigma)} \tilde{L}$ into (17) with equality, we have $\frac{\sigma}{2(1-\sigma)} \tilde{L}=$ $\bar{u}-\frac{p}{\Delta} c$. Then, by Assumption 1-(iv), we have $\frac{c}{\Delta}+\frac{\sigma}{2(1-\sigma)} \tilde{L} \geq \frac{2 c}{\Delta}=b^{*}$. Since $b^{* *}$ is decreasing in $L$, we have $b^{* *}>b^{*}$ for every $L<\tilde{L}$, which also implies $b^{* *}>b^{*}$ at $L<L^{* *}$, and thus $L^{*}<L^{* *}$.

We next find which of two contracts, $\left(f^{*}, b^{*}\right)$ and $\left(f^{* *}, b^{* *}\right)$, yields higher payoff to the principal. The former contract $\left(f^{*}, b^{*}\right)$ yields

$$
(p+\Delta) R+(1-(p+\Delta))\left[\frac{1}{2} \sigma R+\frac{1}{2} r\right]-c-\bar{u},
$$

while the latter contract $\left(f^{* *}, b^{* *}\right)$ yields

$$
(p+\Delta) R+(1-(p+\Delta))\left[\frac{1}{2}\left(\gamma^{*} \sigma R+\left(1-\gamma^{*}\right) r+\frac{1}{2}\left(\lambda^{*} \gamma^{*}(1-\sigma) R+\left(1-\lambda^{*} \gamma^{*}\right) r\right)\right)\right]-c-\bar{u}
$$

Since $\sigma R>r>(1-\sigma) R$ from (2), one can find that $\left(f^{*}, b^{*}\right)$ yields higher payoff to the principal than the other.

Q.E.D. 


\section{References}

Bergemann, Dirk and Martin Pesendorfer (2007), "Information Structures in Optimal Auctions," Journal of Economic Theory, 137(1), 580-609.

Bolton, Patrick, Tano Santos, and Jose A. Scheinkman (2011), "Outside and Inside Liquidity,” The Quarterly Journal of Economics, 126(1), 259-321.

Cai, Li, Chris Cheng Jiang, and Marat Molyboga (2017), “The Moral Hazard Problem in Hedge Funds: A Study of Commodity Trading Advisors," The Journal of Portfolio Management, 43(2), 77-89.

Celik, Gorkem (2009), "Mechanism Design with Collusive Supervision," Journal of Economic Theory, 144(1), 69-95.

Cho, In-Koo and Joel Sobel (1990), "Strategic Stability and Uniqueness in Signaling Games," Journal of Economic Theory, 50(2), 381-413.

Corgnet, Brice and Ismael Rodriguez-Lara (2013), “Are You a Good Employee or Simply a Good Guy? Influence Costs and Contract Design," Journal of Economic Behavior \& Organization, 92, 259-272.

Crawford, Vincent P. and Joel Sobel (1982), "Strategic Information Transmission," Econometrica, 1431-1451.

Eső, Péter and Balazs Szentes (2007), “Optimal Information Disclosure in Auctions and the Handicap Auction,” The Review of Economic Studies, 74(3), 705-731.

Faure-Grimaud, Antoine, Jean-Jacques Laffont, and David Martimort (2003), "Collusion, Delegation and Supervision with Soft Information," The Review of Economic Studies, $70(2), 253-279$.

Ganuza, Juan-José and Jose S. Penalva (2010), "Signal Orderings Based on Dispersion and the Supply of Private Information in Auctions,” Econometrica, 78(3), 1007-1030.

Healy, Paul M. and Krishna G. Palepu (2003), “The Fall of Enron,” Journal of Economic Perspectives, 17(2), 3-26.

Holmstrom, Bengt (1979), "Moral Hazard and Observability," Bell Journal of Economics, 10(1), 74-91.

Holmström, Bengt and Jean Tirole (2011), Inside and Outside Liquidity, MIT press.

Kartik, Navin (2009), "Strategic Communication with Lying Costs," The Review of Economic Studies, 76(4), 1359-1395.

Metrick, Andrew and Ayako Yasuda (2010), "The Economics of Private Equity Funds," The Review of Financial Studies, 23(6), 2303-2341.

Rhee, Keeyoung (2019), “A Study on Delegation Management of Investment in Alternative Assets for NPS Funds,” KDI Policy Study, forthcoming.

Roger, Guillaume (2013), “Optimal Contract under Moral Hazard with Soft Information,” American Economic Journal: Microeconomics, 5(4), 55-80. 